\title{
DVBS2 System Using SDR in Hardware-in-Loop Mode
}

\author{
Soumyasree Bera and Samarendra Nath Sur
}

Sikkim Manipal Institute of Technology, Majitar, Rangpo, Sikkim 737136, India

\begin{abstract}
Digital Video Broadcasting- Satellite $2^{\text {nd }}$ generation (DVBS2) is one of the popular source of entertainment in today's world. It has made TV viewing superb experience due to its ability of sharing high quality picture (i.e. High Definition). But the satellite channel basically follows AWGN type of response as there is lesser chances of multipath generation. The following paper deals with the implementation of the DVBS2 system in Software Defined Radio (SDR) platform and further analysis of its performance both in terms of bit error rate (BER) and actual multimedia transfer capability over different channel conditions like AWGN, and also under multipath condition i.e. Rician channel. All the analysis in the paper is done in hardware-in-loop mode.
\end{abstract}

Index Terms: DVBS2, AWGN, Hardware-In-Loop, BER, SDR, Rician channel

(C) 2017 Published by MECS Publisher. Selection and/or peer review under responsibility of the Research Association of Modern Education and Computer Science

\section{Introduction}

The DVBS2 technology, popular among millions of users, is considered as the standard for digital television throughout the world because of its quality transmission in comparison with cable/terrestrial television transmission. Also in remote places like hilly regions, it's has gained wide popularity.

The system has been designed for different types of applications [1]:

— broadcasting of standard definition and high-definition TV (SDTV and HDTV);

-interactive Services, including Internet access, for consumer applications (for integrated receivers decoders (IRDs) and personal computers);

- professional applications, such as digital TV contribution and news gathering;

— data content distribution and Internet trunking.

The interesting characteristic of DVBS2 is the variation of channel coding and modulation techniques depending on the signal-to-noise ratio that the receiver faces. Low density parity check (LDPC) along with

* Corresponding author. Tel.: +919903884821; fax:

E-mail address: soumyasree.bera@gmail.com 
$\mathrm{BCH}$ makes the system versatile and immune to the channel impairment and can provide $2.9 \mathrm{~dB}$ coding gain compared with Convolution Code at a BER of 10-4 [2]. Also modulations like QPSK, PSK, APSK enhances the system performance in terms of achieving high data rate required for high quality data transmission. It has been specified around three concepts: best transmission performance approaching the Shannon limit, total flexibility, and reasonable receiver complexity [3-6]. Along with the variable coding and modulation, DBV also supports adaptive coding and modulation for one-to-one links that allows transmission parameters optimization depending on the path conditions for each individual user.

Currently DVBS2 is operational at X-Band but Ka band seems to be more to the attractive band of operation of satellite communication due to its huge advantage over X-Band. But Ka-Band has a higher rain attenuation factor which requires further enhancement in system level to cater for the same [7]. Further advancement in DVBS2 is now being considered for an application like satellite to mobile devices communication, where the receiver will be inside railway coach so that passengers can enjoy TV while on move also [8]. Hence such applications includes more challenging channel environment rather than a simple AWGN channel condition.

The authors are aiming for the development of ground based one-to-one communication link which can support real time multimedia communication. So before going for the actual open field test the proposed system is being tested in Hardware-in-the-Loop (HIL) mode. It is a technique that is used for the development and testing of systems which are used for the operation of complex machines and systems. With HIL the physical part of a machine or system is replaced by simulation like environment. This paper gives an introduction to Hardware-in-the-Loop (HIL) and shows how HIL simulation can be used to successfully test control systems. As a result many errors may occur which are to be solved. To reduce these errors, HIL simulation is now gaining widespread attention. The purpose of HIL is to provide an effective platform for developing and testing real-time embedded systems. Hence it is an intermediate step for the checking the hardware system towards idealistic channel.

The DVBS2 system is being realized using WARP v2 board jointly developed and implemented by Mango Communications, Houston, USA \& Rice University, Canada. Wireless Open-Access Research Platform (WARP) [9] is a scalable and extensible programmable wireless platform, built from the ground up to prototype advanced wireless networks. WARP combines high-performance programmable hardware with an open-source repository of reference designs and support materials.

\subsection{CHANNEL:}

One of the implemented pathloss models is the Two Ray Ground model, normally employed for Line-ofSight (LOS) propagation conditions, which approximates the pathloss as:

$$
\begin{array}{r}
P_{\text {loss }}(d)=10 \log _{10}\left(\frac{d^{2}(4 \pi)^{2}}{\lambda^{2}}\right) \text { if } \quad d<d_{c} \\
10 \log _{10}\left(\frac{d^{4}}{h_{A}{ }^{2} h_{B}{ }^{2}}\right) \text { if } \quad d \geq d_{c}
\end{array}
$$

Where,

$$
d_{c}=\frac{4 \pi h_{A} h_{B}}{\lambda}
$$

and $\mathrm{d}$ is the distance between transmitter and receiver, $\mathrm{hA}$ and $\mathrm{hB}$ are their respective antenna heights, and $\lambda$ is the carrier wavelength, all of them in meter. For $\mathrm{d}<\mathrm{dc}$, this model is equivalent to the Free Space propagation model. In urban environments, one has to consider a log-distance pathloss model, based on the following 
expression:

$$
P_{\text {loss }}(d)=P_{\text {loss }}\left(d_{0}\right)+10 n \log _{10}\left(\frac{d}{d_{0}}\right)
$$

Where $\mathrm{n}$ being the pathloss exponent which is determined based on the experimental results. $P_{\text {loss }}\left(d_{0}\right)$ pathloss experienced at reference distance d0. $P_{\text {loss }}\left(d_{0}\right)$ can be calculated using the free space propagation model.

\section{- AWGN channel:}

Additive White Gaussian Noise is the basic type of linear channel model which performs additive operation of wideband white noise having constant spectral density and a Gaussian distribution of amplitude which is the ideal channel for satellite and space communication. The relationship between Es/N0 and Eb/N0, both expressed in $\mathrm{dB}$, is as follows:

$$
\text { Es/N0 }(\mathrm{dB})=\mathrm{Eb} / \mathrm{N} 0(\mathrm{~dB})+10 \log 10(\mathrm{k})
$$

where $\mathrm{k}$ is the number of information bits per symbol.

\section{- $\quad$ Rice Channel:}

Rician fading takes when the line of sight signal, is much stronger than the multipath. Rician distribution is used to characterize the amplitude gain. The parameters used to characterize the Rician channel are K and $\Omega$. K is the ratio between the power in the LOS path and the power in the multipath. $\Omega$ is the total power all the paths, i.e., $\Omega=v^{2}+\sigma^{2} . \Omega$ is the scaling factor.

The amplitude of the signal received $\mathrm{R}$ is then distributed with parameters

$$
v^{2}=\frac{k}{k+1} ; \sigma^{2}=\frac{\Omega}{2(1+k)}
$$

Hence the probability distributed function of the Rician channel is-

$$
f(x)=\frac{2(k+1) x}{\Omega} \exp \left(-k-\frac{(k+1) x^{2}}{\Omega}\right) \mathrm{I}_{0}\left(2 \sqrt{\frac{k(k+1) x}{\Omega}}\right)
$$

Where $\mathrm{I}_{0}()$ is the 0th order modified Bessel function of the first kind.

$$
P_{b}=0.5 \operatorname{erfc}\left[\sqrt{\frac{k(E b / N o)}{(k+E b / N o)}}\right]
$$

where $\mathrm{Pb}$ is the probability of error; Eb is the energy per bit; $\mathrm{NO}$ is the noise power. 


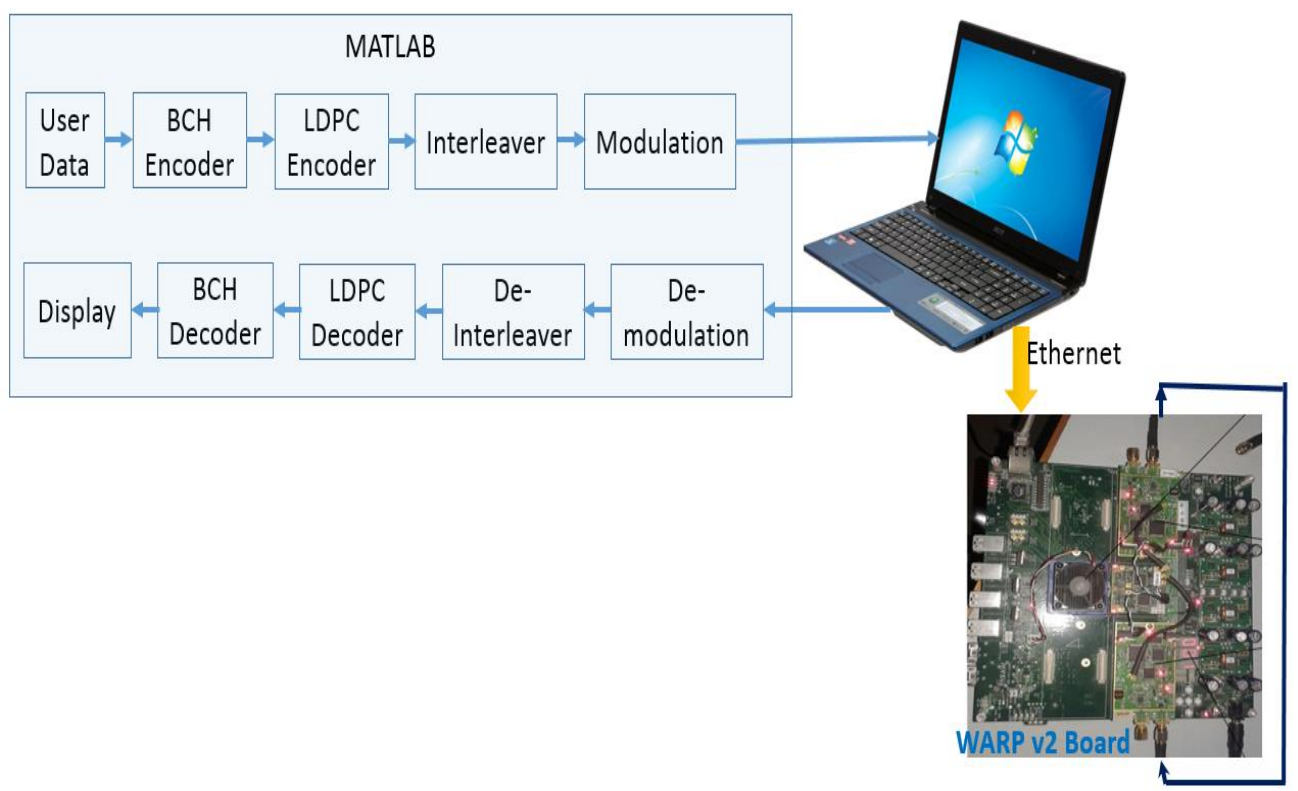

Fig.1. Total Hardware System Setup

Table 1. System Specification

\begin{tabular}{lll}
\hline S1. No. & Parameter & Value \\
\hline 1 & Cable length & $0.5 \mathrm{~m}$ \\
2 & RF Frequency & $2.462 \mathrm{GHz}$ \\
3 & TX Amp Gain & $1 \mathrm{~dB}$ \\
4 & RX RF gain & $0 \mathrm{~dB}$ \\
5 & RX BB Gain & $20 \mathrm{~dB}$ \\
6 & SDR used & WARP v2 board \\
\hline
\end{tabular}

The system specifications are shown on table 1. Rather than going directly from simulation to open range, the transmitter and the receiver is connected via a cable of length $0.5 \mathrm{~m}$ as seen in Fig 1 and different performance analysis has been done.

\section{Results}

\subsection{BER Performance:}

At first the system, is tested in terms of its BER performance. Under different channel conditions the BER performance is verified and with variable modulation, code rate. Also the system is tested under AWGN and also different Rician factor channel.

i) System Consideration:

- Channel: wired channel

- Code rate: $1 / 2$

- Modulation: variable 
As can be seen from fig 2, that BPSK performs best under low SNR condition. BPSK has nearly 10dB advantage over QPSK. So at lower SNR, BPSK is more preferable than QPSK but data rate wise QPSK is better.

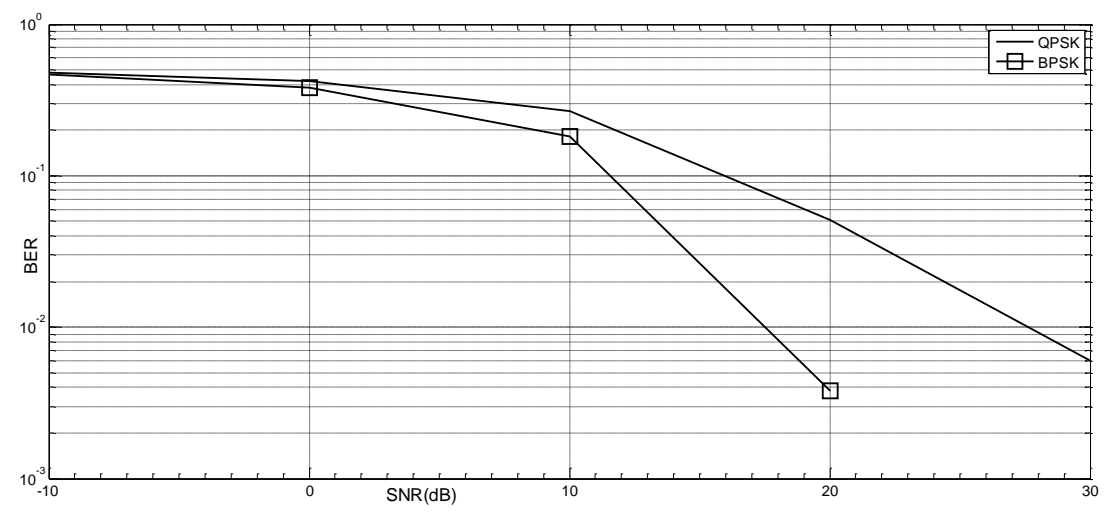

Fig.2. BER vs SNR performance of DVBS2 system under different modulation type

ii) System Consideration:

- Channel: Rician

- Code rate: $1 / 2$

- Modulation: BPSK

- Rician K-Factor: variable

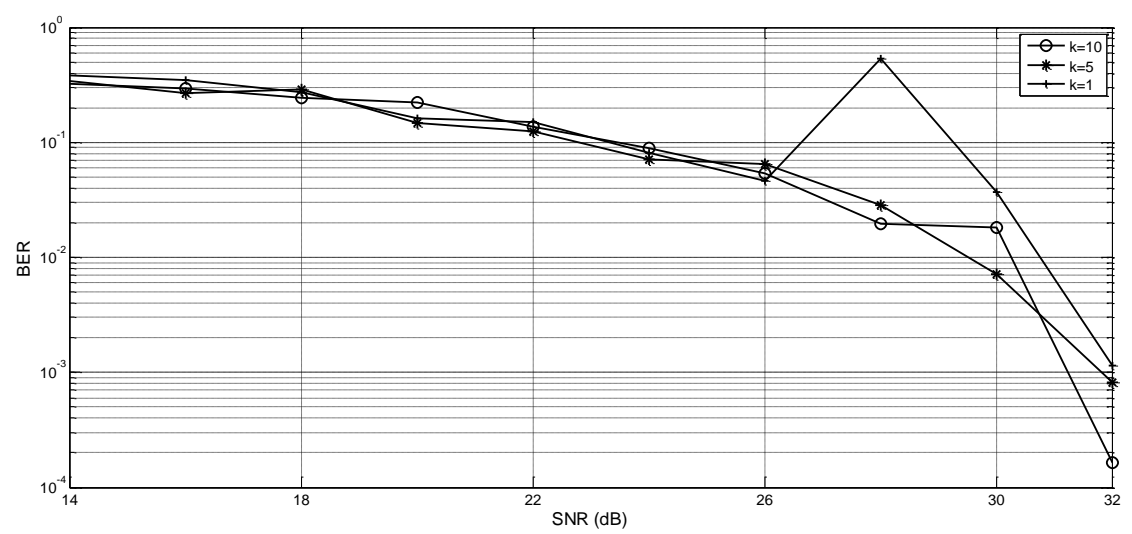

Fig.3. BER vs SNR performance of DVBS2 system under different Rician channel condition with BPSK modulation

Fig 3 represents the performance of the developed DVBS2 system under Rician channel condition and at K factor with BPSK modulation. $\mathrm{K}=1$ is the severe condition in the channel and so the system has the worst performance. The same test is repeated for QPSK modulation (Fig. 4) and when compared, the performance of the system is best under BPSK, $\mathrm{K}=10$.

iii) System Consideration:

- Channel: Rician

- Code rate: $1 / 2$ 
- Modulation: QPSK

- Rician K-Factor: variable

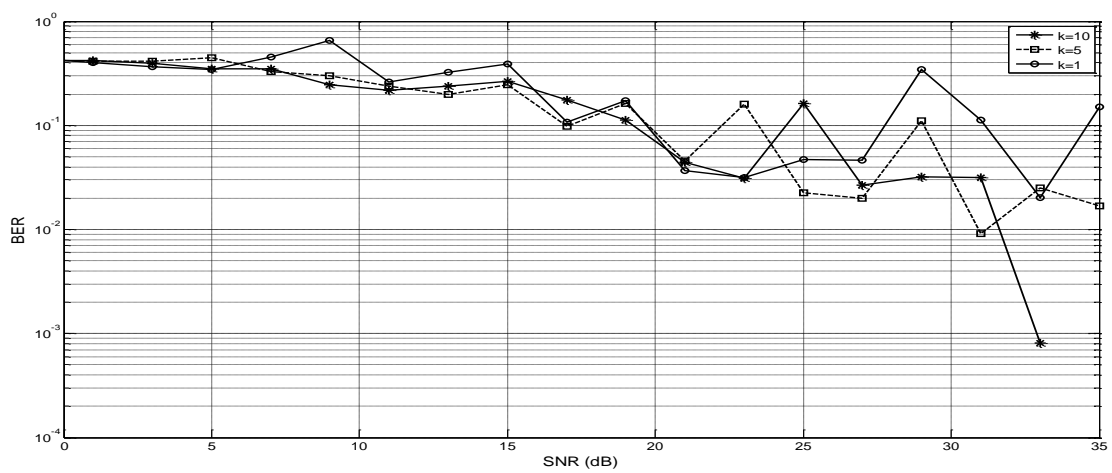

Fig.4. BER vs SNR performance of DVBS2 system under different Rician channel condition with QPSK modulation

Therefore, the BER achieved by the system under rice channel is nearly 1E-4 with BPSK modulation. The next step followed after BER verification is the actual data transfer i.e. audio, text, image etc. Hence in the next article stress has been given on whether the system is able to perform well when user data is being communicated.

\subsection{Multimedia Communication:}

Multimedia communication capability is the ultimate goal of the developed system. Therefore the user data is changed to multimedia data like image. But before the system is tested for communicating image data, the system is characterized by its performance for simpler multimedia data i.e. tone. Furthermore the system verified with image data transfer after the successful reception of tone.

a) Tone Test:

In order to verify the system performance over tone, the user data is taken as $1 \mathrm{KHz}$ sinusoidal signal. This data is DVBS2 encoded and transmitted. The received signal is then passed through the DVBS2 decoder and processed. The decoded received user data is then tapped from the audio port of the PC and its equivalent frequency domain information is being captured. Fig 5(a) shows the transmitted signal spectrum.

System Consideration:

- Code rate: $1 / 2$

- Modulation: QPSK

- User data: $1 \mathrm{KHz}$ sine wave 


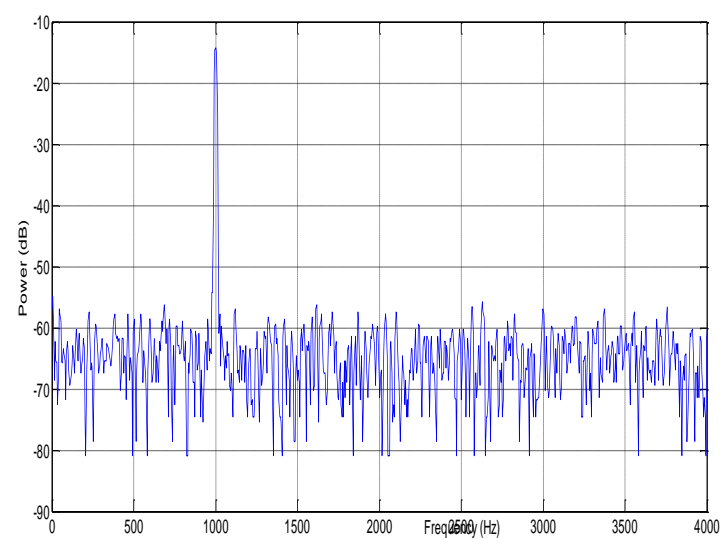

(a)

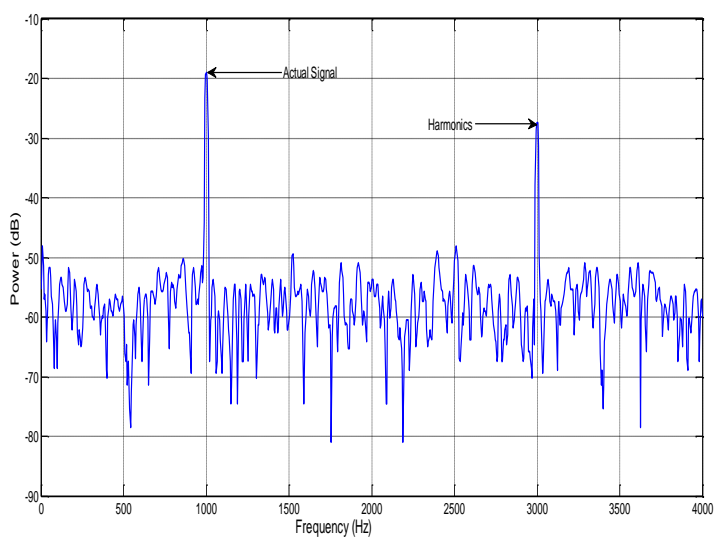

(b)

Fig.5(a). Spectrum of $1 \mathrm{KHz}$ (b) Spectrum of $1 \mathrm{KHz}$ transmitted tone received tone

As can be seen that the received signal Fig 5(b) receives the $1 \mathrm{KHz}$ signal as well as its harmonics due to carrier frequency offset (CFO) which is may by applying the offset removal technique.

b) Image Transfer:

The next test criteria is for image. Hence an image data is transmitted and received.

System Consideration:

- Code rate: $1 / 2$

- Modulation: QPSK

- User data: Image file
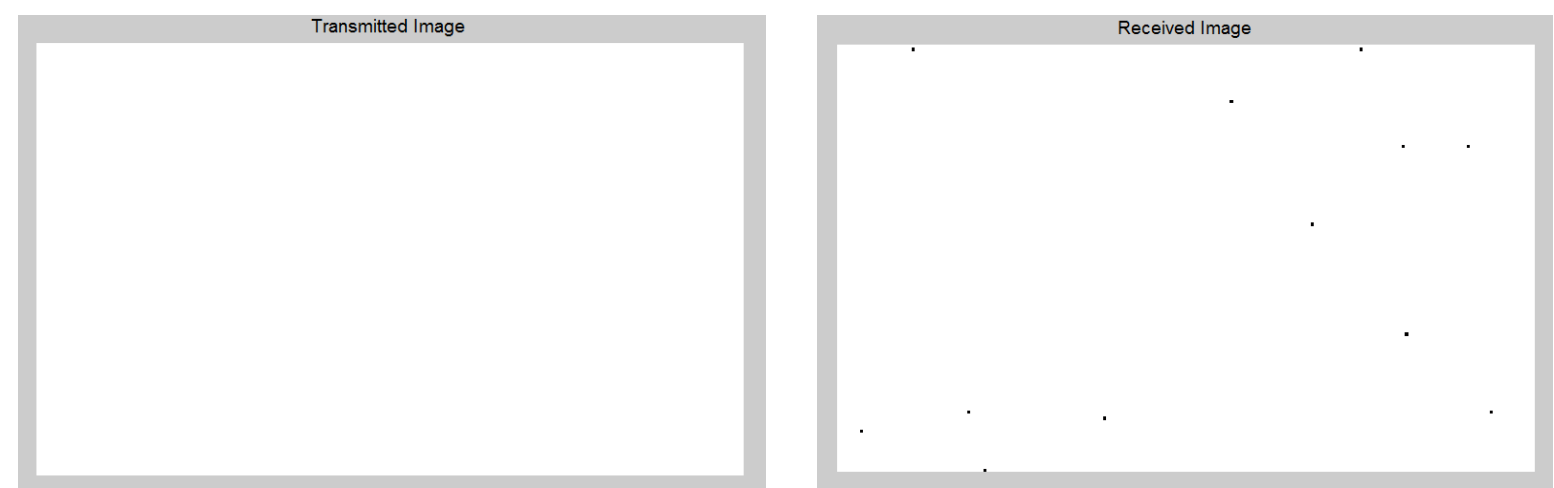

(a)

(b)

Fig.6. (a) Image Transmission (b) Image reception

For image test, the simplest of image (Fig 6(a)) is taken and being transmitted. The received image (Fig 6(b)) is being retrieved but as can be noted that there are some black spot which is due to the presence of noise in between TX and RX chain. 


\section{Conclusions}

DVB technology is widely used technique but limited to satellite communication till date. The same is be extended for ground based wireless communication and it shows a promising result towards this effort. The use of LDPC code plays a greatly under low SNR condition. Also the use of higher modulation technique leads the system capacity closure to the Shannon's limit. The same system is used to transfer image under different channel conditions. The developed model is tested on actual hardware over closed loop (HIL) mode. At first the ber performance is performed under different rician channel conditions also for different modulation also. Under good channel condition, ber of 1E-04 achieved at high SNR. Also the system works well under rician channel i.e. ber of 1E-03@ $\mathrm{K}=1$. After BER performance verification, the system testing is done with multimedia data like tone, image etc. In case of tone transfer, harmonics are generated in the received signal. Hence harmonics removal techniques to be applied for more accuracy in the received signal. In case of image transfer also, the received image is having some granular noise which is undesirable. The authors are working further in order to remove the above said limitations and also trying to test the performance of the system under far severe channel conditions.

\section{References}

[1] A Morello and V Mignone, "DVB-S2: The Second Generation Standard for Satellite Broad-band Services," Proceedings of the IEEE, Vol. 94, No. 1, pp-210-227, January 2006.

[2] D.Xinyu, W.Yanling and X.Tiecheng, "Performance of LDPC Codes for Satellite Communication in Ka Band," Proceedings of the 5th International Conference on Wireless communications, networking and mobile computing, WiCOM, pp- 715-718, 2009.

[3] ETSI EN 302307 V1.2.1 (2009-08) European Standard (Telecommunications series) Digital Video Broadcasting (DVB); Second generation framing structure, channel coding and modulation systems for Broadcasting, Interactive Services, News Gathering and other broadband satellite applications (DVB-S2).

[4] EN 300421 v1.1.2: Digital Video Broadcasting (DVB); Framing structure, channel coding and modulation for $11 / 12 \mathrm{GHz}$ satellite services.

[5] ETSI EN 301790 v1.4.1: Digital Video Broadcasting (DVB): Interaction channel for satellite distribution systems.

[6] ETSI TR 101790 v1.3.1: Digital Video Broadcasting (DVB): Interaction channel for satellite distribution systems; Guidelines for the use of EN 301790.

[7] P.Angeletti and M.Lisi, "A Systemic Approach to the Compensation of Rain Attenuation in Ka-Band Communication Satellites," International Journal of Microwave Science and Technology, Volume 2012, pp- 1-7, 2012.

[8] M. Iubatti, G. E. Corazza , A. Vanelli-Coralli and M. Villanti "DVB-S2 in Railway Environments: An Exploratory Study for Tunnel Coverage," International Workshop on Satellite and Space Communications, pp- 71-75, 14-15 September, 2006.

[9] https://warpproject.org/trac/wiki/HardwareUsersGuides/FPGABoard_v2.2 


\section{Authors' Profiles}

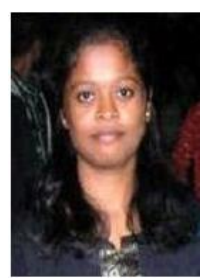

Soumyasree Bera: Born in 1988 at Kolaghat, West Bengal, INDIA. She received her M. Tech from Sikkim Manipal Institute of Technology, Sikkim Manipal University, in the year 2015. Currently she is working as Faculty in the Department of Electronics \& Communication Engineering, in same college. Her area of Interest includes Remote Sensing, Wireless Communication, Advanced Digital Signal Processing.



Samarendra Nath Sur: Born in 1984 at Hooghly, WestBengal, INDIA. Received M.Sc. (Electronics Science) from Jadavpur University in the year 2007, M.Tech from Sikkim Manipal University and persuing PhD from NIT, Durgapur. Currently working as Asst Professor, E\&C Dept, Sikkim Manipal University, India. Broadband Wireless Communication, Remote Sensing are the area of specializations

How to cite this paper: Soumyasree Bera, Samarendra Nath Sur,"DVBS2 System Using SDR in Hardware-inLoop Mode", International Journal of Wireless and Microwave Technologies(IJWMT), Vol.7, No.2, pp.35-43, 2017.DOI: 10.5815/ijwmt.2017.02.04 